\title{
3D Acoustic Field Intensity Probe Design and Measurements
}

\author{
Józef KOTUS(1),(2), Andrzej CZYŻEWSKI ${ }^{(1)}$, Bożena KOSTEK ${ }^{(2)}$ \\ (1) Multimedia Systems Department \\ Faculty of Electronics, Telecommunications and Informatics \\ Gdańsk University of Technology \\ Narutowicza 11/12, 80-233 Gdańsk, Poland; e-mail: \{joseph, andcz\}@sound.eti.pg.gda.pl \\ (2) Audio Acoustic Laboratory \\ Faculty of Electronics, Telecommunications and Informatics \\ Gdańsk University of Technology \\ Narutowicza 11/12, 80-233 Gdańsk, Poland; e-mail: bokostek@audioakustyka.org
}

(received April 5, 2016; accepted July 12, 2016)

\begin{abstract}
The aim of this paper is two-fold. First, some basic notions on acoustic field intensity and its measurement are shortly recalled. Then, the equipment and the measurement procedure used in the sound intensity in the performed research study are described. The second goal is to present details of the design of the engineered 3D intensity probe, as well as the algorithms developed and applied for that purpose. Results of the intensity probe measurements along with the calibration procedure are then contained and discussed. Comparison between the engineered and the reference commercial probe confirms that the designed construction is applicable to the sound field intensity measurements with a sufficient effectiveness.
\end{abstract}

Keywords: sound intensity; acoustic vector sensor; calibration; measurements.

\section{Introduction}

Sound intensity is a measure of the flow of acoustic energy in a sound field. More precisely, the sound intensity $I$ is a vector quantity defined as the time average of the flow of sound energy through a unit area in a direction perpendicular to the area. The intensity in a certain direction is the product of sound pressure (scalar) $p(t)$ and the particle velocity (vector) component in that direction $u(t)$. The SI units for sound intensity are Watts per square meter $\left(\mathrm{W} / \mathrm{m}^{2}\right)$. Although acousticians have attempted to measure this quantity since the 1930s, the first reliable measurements of sound intensity under laboratory conditions did not occur until mid-1970s. Commercial sound intensity measurement systems were introduced to the market in early 1980s, and the first international standards for sound intensity measurements and related instruments were issued in the mid-1990s (JACOBSEN, 2008; 2011; IEC, 1993; ANSI, 1996; De BREE, 2003; FAнY, 1995).

Typically, intensity metric quantities are used for measuring energy transmission and propagation paths
(WEYNA, 2003; 2010), as well as for detection of noise source localization (Kotus, 2015), determination of acoustic impedance and reflection index of materials (De Bree, 2003; Weyna, 2003), although one may find several examples of employing them in audio engineering (Cengarle, Mateos, 2011; WoszczyK et al., 2007), and in particular in the recording and reconstruction of the acoustic field, e.g. ambisonics (Cengarle et al., 2011). An interesting application of sound intensity technique for analysis and visualization of directional room responses was presented in 2001 during the 111th AES Convention in USA (MerimaA et al., 2001). The authors applied a 3D microphone array for intensity calculation $(p-p$ intensity probe) for directional analysis of the measured room responses. Since impulse responses are inherently multidimensional, thus they introduced a new way of visualizing 5-dimensional data, and showed its usefulness in demonstrating time-frequency properties of the responses (MERIMAa et al., 2001). An interesting but still unexplored approach is the sound intensity measurement technique applied to gunshot detection (Aguilar, 2015). Also, such a technique is used in 
the car audio system measurements as in a study by TERVo et al. (2015).

Numerous research investigations involving the study of the distribution of acoustic energy around the human head and directional sound radiation of the selected musical instruments were also performed by the authors of this study using sound intensity technique (Kotus, Kostek, 2015; Kotus et al., 2015a; 2015b).

Sound intensity measurement systems produced commercially today, with the exception of Microflown's Ultimate Sound Probes, are based on the 'twomicrophone' (or ' $p-p$ ') principle, which uses two closely spaced pressure microphones and rely on a finite difference approximation of the sound pressure gradient. The IEC 1043 standard on instruments for the measurement of sound intensity, which was published in 1993, deals exclusively with the $p-p$ measurement principle (JACOBSEN, 2008; 2011; IEC, 1993; ANSI, 1996; DE BREE, 2003; FAHY, 1995).

The time-averaged intensity $I$ in a single direction is given by Eq. (1):

$$
I=\frac{1}{T} \int_{T} p(t) u(t) \mathrm{d} t,
$$

where $p(t)$ is the acoustic pressure (scalar) and the $u(t)$ is the particle velocity (vector) component. The $p-p$ measurement principle employs two closely spaced pressure microphones. The particle velocity is obtained through the Euler's relation, Eq. (2), as in (JACOBSEN, 2008):

$$
\widehat{u}_{r}(t)=-\int_{-\infty}^{t} \frac{p_{2}(\tau)-p_{1}(\tau)}{\rho \Delta r} \mathrm{~d} \tau,
$$

where $p_{1}$ and $p_{2}$ are the sound pressure signals from the two microphones, $\Delta r$ is the microphone separation distance, $\rho$ is the density of the medium and $\tau$ is a dummy time variable. The caret indicates the finite difference estimate, which is an approximation of the real sound pressure gradient (JACOBSEN, 2008). The sound pressure at the center of the probe is estimated as the average value for both microphones.

The sound intensity probes are rather expensive. Consequently, the main objective of the presented work was to design and evaluate the $3 \mathrm{D}$ intensity probe using inexpensive components.

The Sound Intensity Probe (3D SIP), proposed by the authors, uses widely available, low-cost components. Determining the sound intensity is based on the analysis of sound pressure gradient, consequently this is the $p-p$ type of probe. The value of the sound intensity corresponding to the pressure gradient is determined by an additional microphone located centrally between the microphones forming each pair of $p-p$ probes. These microphones are arranged in a rectangular pattern, thus the determined intensity components can easily be visualized using Cartesian coordinates.
By definition, the $p-p$ type intensity probe can only operate within a limited frequency range. This limitation is a direct result of the probe design, in particular the distance between the paired $p p$ microphones. According to formula (2), a component related to the particle velocity is determined as the difference between the sound pressure for a couple of microphones. The value of this difference is strongly dependent on the wavelength or, which is equivalent, on the frequency of the signal. Figure 1 shows a set of characteristics representing the shapes of sine waves at a given frequency as a function of distance. The fundamental frequency was $128 \mathrm{~Hz}$. The frequency of individual waveforms was determined at the interval of 1/12 octave. There are 75 waveforms shapes presented with frequencies ranging from $136 \mathrm{~Hz}$ to $9742 \mathrm{~Hz}$. The waveform line color, from red to purple, corresponds to a given frequency. In addition, a vertical black line marks the distance between the paired $p-p$ microphones designed and tested in the sound intensity probe. The clearly visible relationship is that for low frequencies, the difference between the amplitude values at the 0 and $12 \mathrm{~mm}$ points is very small. This difference increases as the frequency increases. The consequence of this is low accuracy and sensitivity of the $p-p$ probes for low frequencies and at frequencies with wavelength shorter than the distance between microphones. This means that the distance between the microphones directly determines the frequency range for which the particle velocity can properly be evaluated. To ensure proper operation of the $p-p$ probe, the non-
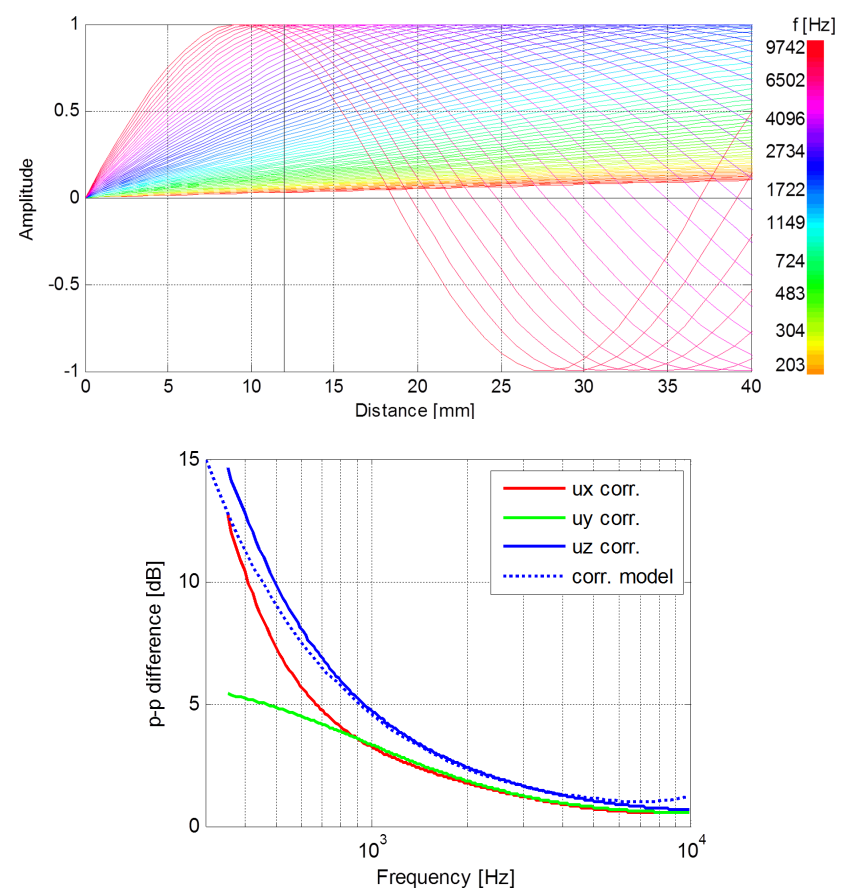

Fig. 1. Distribution of amplitude as a function of the distance between microphones, a black vertical line indicates a $12 \mathrm{~mm}$ distance, as is in the developed sound intensity probe. 
linear dependence of the sensitivity of the frequency requires compensation. The theoretical shape of the probe sensitivity compensation characteristics can be obtained by calculating the inverse of the difference of the sound pressure over a length corresponding to the distance between the pair of $p-p$ microphones in the function of frequency. The characteristics are presented in the form of a dotted line marked in blue on the right diagram in Fig. 1. The graph also shows the actual compensation characteristics determined by measurements made for the 3D SIP probe. The compatibility of shapes and values for the curve correcting the $p-p$ pair in planes $z$ and $x$ is clearly visible. Some discrepancies may be observed for a $p-p$ pair acting in the plane $y$. The consequences of the disclosed discrepancies are discussed in Sec. 3. The process of determining the individual characteristics of the compensation constraints arising from the probe structure and properties of the microphones are described in detail in Sec. 3, in particular in Section entitled Calibration (Subsec. 2.3).

\section{3D-intensity probe design}

The designed 3D sound intensity probe is based on the two functional components: hardware consisting of microphones and a conditioning module and software responsible for the amplitude and phase correction and calculation. All parts of the probe were designed and assembled by the authors. The complete intensity probe is presented in Fig. 2. The probe delivers four analogue signals: acoustic pressure (additional microphone in the middle of the probe) and the air particle velocity signals obtained employing the pairs of two closely spaced pressure microphones. Output sig-

a)

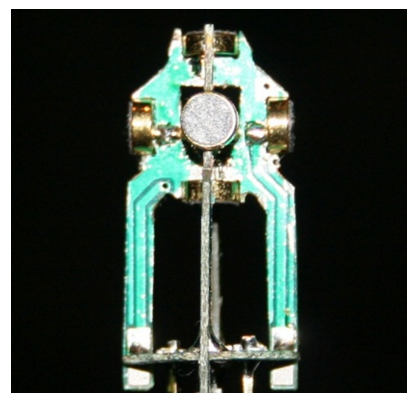

b)

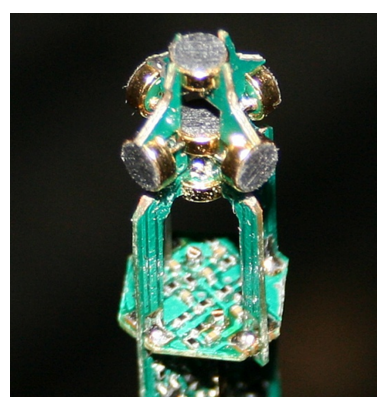

Fig. 2. 3D SIP in different orientations, all microphones are visible. nals are converted into the digital form using four channels sound card (MAYA Sound CARD, 2016). Next, the signals are processed in the digital domain. Additional details are presented below.

\subsection{Hardware part}

The miniature microphones are applied in the presented design. A single microphone has a diameter of $4 \mathrm{~mm}$ and a height of $1.5 \mathrm{~mm}$ (type: KEEG1542PBL delivered by KINGSTATE). The space between the microphones in a single pair is equal to $12 \mathrm{~mm}$. All pairs of microphones are carefully selected on the basis of their frequency responses.

\subsection{Software part}

The block diagram of the algorithm applied for the sound intensity determination is depicted in Fig. 3. Sound intensity calculation algorithm required signals delivered by the multichannel acoustic vector sensor. It produced the following signals: sound pressure $p$ and three orthogonal particle velocity components $u_{x}$, $u_{y}, u_{z}$.

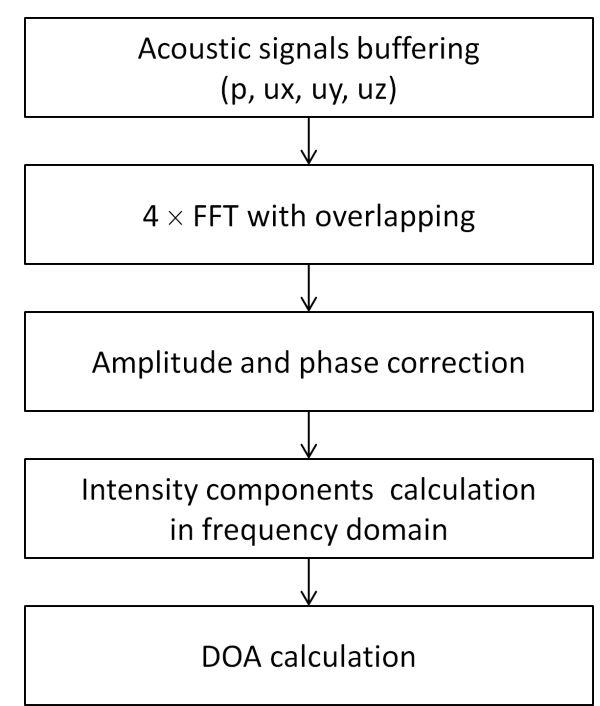

Fig. 3. The block diagram of the algorithm applied for the sound intensity determination.

After triggering the calculation by the synchronization signal (see Subsec. 3.2 for details), the FFT calculation for measurement signals was performed. The Hanning window was applied. Subsequently, the 4096 point FFT calculation for each signal was performed with the sampling frequency equal to $48 \mathrm{kS} / \mathrm{s}$ (frequency resolution: $10.8 \mathrm{~Hz}$ ). Such parameters provide sufficient spectral resolution for sound intensity calculations. The overlap was equal to $50 \%$. The FFT calculation was performed for each acoustic component $\left(p, u_{x}, u_{y}, u_{z}\right)$, separately. This operation yields transformed signals: $X_{p}(i), X_{u x}(i), X_{u y}(i), X_{u z}(i)$ where $i$ 
(ranging from 0 to 4095) denotes the index of the spectral bin.

The matrix X (Eq. (3)) contains information about the arrival direction for every spectral component of the signal

or

$$
\mathbf{X}=\left[\begin{array}{llll}
X_{p}(i) & X_{u x}(i) & X_{u y}(i) & X_{u y}(i)
\end{array}\right]
$$

$\mathbf{X}=\left[\Im\{p(n)\} \Im\left\{u_{x}(n)\right\} \Im\left\{u_{y}(n)\right\} \Im\left\{u_{z}(n)\right\}\right]$.

The sound intensity vector is defined and calculated according to Eq. (5).

$$
\mathbf{I}(i)=\left[\begin{array}{c}
I_{x}(i) \\
I_{y}(i) \\
I_{z}(i)
\end{array}\right]=\left[\begin{array}{c}
X_{p}(i) \cdot \overline{X_{u x}(i)} \\
X_{p}(i) \cdot \overline{X_{u y}(i)} \\
X_{p}(i) \cdot \overline{X_{u z}(i)}
\end{array}\right],
$$

where $I_{x}(i)$ - sound intensity component for $x$ direction for $i$-th spectral components, $X_{p}(i)$ - coefficients of complex spectrum for $i$-th spectral components for acoustic pressure signal, $\overline{X_{u x}(i)}$ - conjugated spectrum coefficients for particle velocity in $x$ direction.

On the basis of signals delivered by the probe the sound intensity components and the direction of arrival (DOA) data can be determined. For proper probe operation, the correction of the amplitude and phase for the particle velocity signals was required.

\subsection{Calibration}

The calibration process was performed in an anechoic chamber. The probe was placed in front of a loudspeaker at 1 meter distance, in front of the speaker. In such position we can observe signals of acoustic pressure $p(t)$ and particle velocity $v(t)$ in phase. Moreover the relation between such signals in the considered position should be constant. It depends on the properties of the propagation medium and it is called acoustic impedance $\left(Z_{a}\right)$ and can be written as:

$$
Z_{a}=\frac{p(t)}{v(t)}=\rho c
$$

where $\rho$ is the density of the medium, $c$ is the speed of sound.

The main aim of the calibration process is to measure the discrepancies between the acoustic pressure and particle velocity in the considered direction. In the first phase, the $3 \mathrm{D}$ SIP $u_{x} p-p$ pair was directed to the speaker. Next the sensor was rotated manually to prepare a calibration of other $p-p$ pairs $\left(u_{y}\right.$ and $\left.u_{z}\right)$. This way, each $p-p$ pair was calibrated separately. The coaxial loudspeaker was applied as a sound source (Coax Studio Monitor, 2016). A chirp signal in frequency range from $100 \mathrm{~Hz}$ up to $10000 \mathrm{~Hz}$ over a period of 10 seconds was used. The measured difference characteristics (between acoustic pressure and particle velocity for given direction) were interpolated using the polynomial regression model (STIGLER, 1974). In practice, the $a_{a 0}-a_{a 5}$ coefficients given in Eqs. (6) and (7) can be found using the MATLAB software using polyfit function. Obtained results, both for the amplitude and phase, are shown in Fig. 4. Values obtained on the basis of performed measurements are depicted using a solid line. A dotted line is used to present the characteristic of the calculated model function. The 5th degree polynomial was applied for the amplitude correction function and the 4th degree polynomial for the phase correction function.


Fig. 4. Amplitude correction characteristics (top graph) and phase correction characteristics (botoom graph) for each particle velocity signals.

The general polynomial regression model equations for both the amplitude $\left(y_{c . a m p l, i}\right.$ - the amplitude correction factor, expressed in $\mathrm{dB})(7)$ and phase $\left(y_{c \text {.phase }, i}\right.$ - the phase correction factor, expressed in degrees) (8) are as follows:

$$
\begin{aligned}
y_{c . \text { ampl }, i}= & a_{a 0}+a_{a 1} \cdot x_{i}+a_{a 2} \cdot x_{i}^{2}+a_{a 3} \cdot x_{i}^{3} \\
& +a_{a 4} \cdot x_{i}^{4}+a_{a 5} \cdot x_{i}^{5} \\
y_{c . \text { phase }, i}= & a_{f 0}+a_{f 1} \cdot x_{i}+a_{f 2} \cdot x_{i}^{2}+a_{f 3} \cdot x_{i}^{3} \\
& +a_{f 4} \cdot x_{i}^{4}
\end{aligned}
$$

where $a_{a 0}-a_{a 5}-$ constant values for the amplitude correction, $a_{f 0}-a_{f 4}-$ constant values, $i$ - index of the current FFT bin, $x_{i}$ - value, related to frequency used 
in formulas (7) and (8), is computed according to formula (9):

$$
x_{i}=\left(\log _{10}(i \cdot \Delta f)-2\right) /\left(2 / N_{f}+1\right),
$$

where: $\Delta f$ - frequency resolution of the FFT filtration (in the considered implementation FFT length was equal to 4096 bins, sampling frequency was equal to $48000 \mathrm{Sa} / \mathrm{s}, \Delta f$ was equal to $48000 / 4096), N_{f}$ - number of frequency bins taken into consideration during the calibration process. The value of this parameter can be calculated including the length of the calibration signal, sampling frequency value, FFT length and $50 \%$ overlap factor. In the applied conditions the $N_{f}$ was equal to $(10 \cdot 48000) /(0.5 \cdot 4096)$. Finally, the $N_{f}$ parameter was equal to 234 .

The constant values $a_{a 0}-a_{a 5}$ and $a_{f 0}-a_{f 4}$ were calculated separately for every $p-p$ pair $\left(u_{x}, u_{y}, u_{z}\right)$.

\section{Evaluation of 3D intensity probe}

The developed SIP probe was tested in free field conditions. The USP sensor by Microflown was used as a reference probe. Both sensors were placed in close proximity to each other in the vertical plane. This position of sensors provides an equal distance to the speakers in the horizontal plane.

Four Genelec speakers model 6010A (Genelec Studio Monitor, 2016) were used as sources reproducing acoustic signals, arranged at the distance of 30 degrees in the horizontal plane and in the distance of about 9 degrees in the vertical plane. The location of the speakers is shown schematically in Fig. 5. The left part of Fig. 5 shows a view from the top of the speaker, the right part of Fig. 5 shows a side view. Next to each speaker, their numbers and the approximate value of the angle are provided. The color scheme is consistently applied in the rest of the study for other drawings as well as when presenting the measurement results obtained for each speaker.

Figure 6 shows photographs of the measuring system, illustrating the relative position of sensors (photograph a)) and audio sources (photographs b) and c)). Each speaker was presented using a different color. The

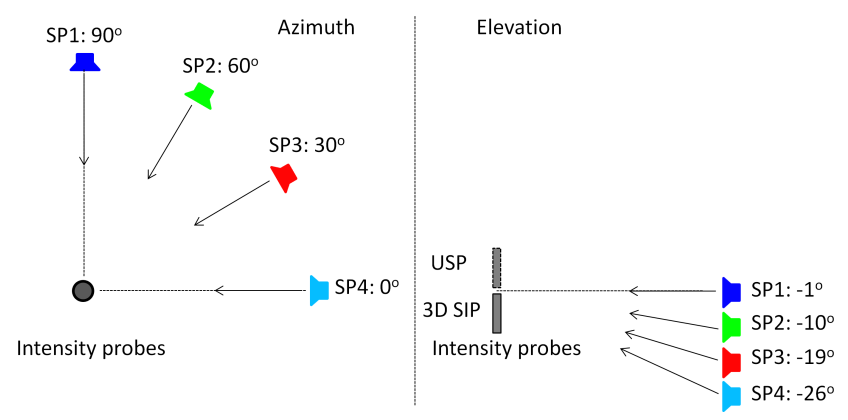

Fig. 5. Measurement setup.

a)

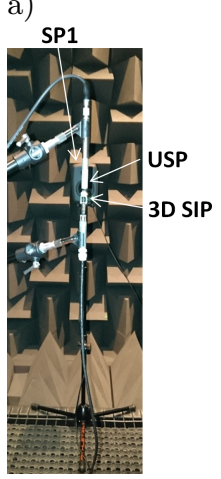

b)

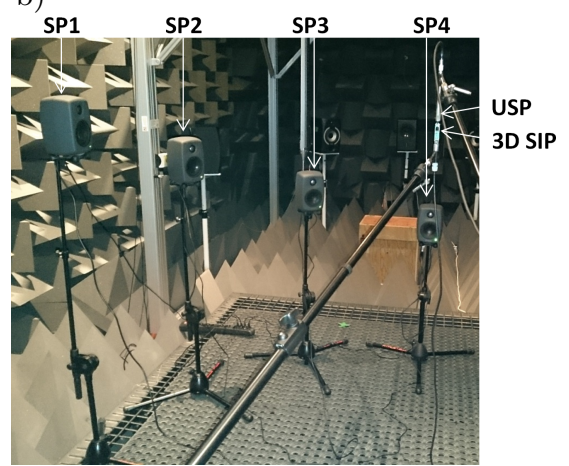

c)

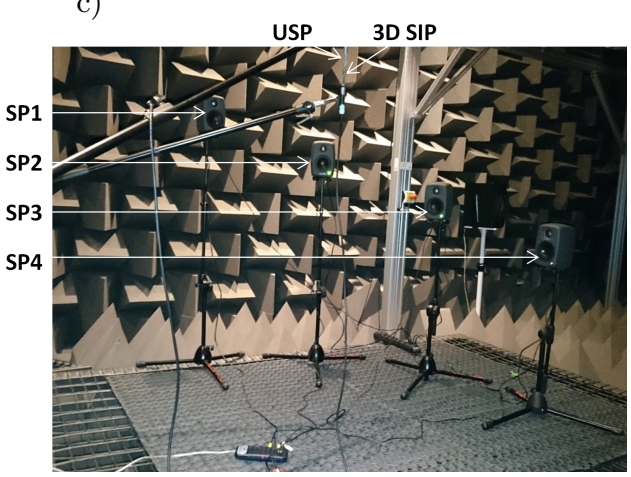

Fig. 6. Measurement setup.

spatial arrangement of speakers is clearly visible in the photographs b) and c). In each of the photographs, the position of the examined intensity probe is indicated.

Table 1 shows the exact data on position of used sensors in relation to each speaker. The reference data

Table 1. Precise information on the location of speakers relative to the probes.

\begin{tabular}{|c|c|c|c|c|c|c|c|c|c|}
\hline \multirow{2}{*}{\multicolumn{2}{|c|}{$\begin{array}{c}\text { Orientation } \\
\text { Speaker number }\end{array}$}} & \multicolumn{4}{|c|}{ Azimuth [deg] } & \multicolumn{4}{|c|}{ Elevation $[\mathrm{deg}]$} \\
\hline & & 1 & 2 & 3 & 4 & 1 & 2 & 3 & 4 \\
\hline \multirow{3}{*}{$3 \mathrm{D} \mathrm{SIP}$} & $\alpha_{\mathrm{GTmin}}$ & 86.7 & 56.7 & 26.7 & -3.3 & 1.4 & -7.8 & -16.2 & -24.3 \\
\hline & $\alpha_{\mathrm{GT}}$ & 90.0 & 60.0 & 30.0 & 0.0 & -0.5 & -9.8 & -17.9 & -25.9 \\
\hline & $\alpha_{\text {GTmax }}$ & 93.3 & 63.3 & 33.3 & 3.3 & -2.5 & -11.7 & -19.7 & -27.5 \\
\hline \multirow{3}{*}{ USP } & $\alpha_{\mathrm{GTmin}}$ & 86.7 & 56.7 & 26.7 & -3.3 & 0.3 & -8.9 & -17.2 & -25.3 \\
\hline & $\alpha_{\mathrm{GT}}$ & 90.0 & 60.0 & 30.0 & 0.0 & -1.6 & -10.9 & -19.0 & -26.8 \\
\hline & $\alpha_{\text {GTmax }}$ & 93.3 & 63.3 & 33.3 & 3.3 & -3.6 & -12.8 & -20.7 & -28.4 \\
\hline \multicolumn{2}{|l|}{$\alpha_{\text {Lim }}$} & \pm 3.3 & \pm 3.3 & \pm 3.3 & \pm 3.3 & \pm 1.9 & \pm 1.9 & \pm 1.8 & \pm 1.6 \\
\hline
\end{tabular}


are shown in degrees. The data are detailed in the plane of azimuth and elevation. Significant differences exist between the 3D SIP sensors and USP for elevation angles. They result from the fact that the USP sensor was $50[\mathrm{~mm}]$ over the 3D SIP sensor. Values in bold are used to determine the sound source location errors. The ground truth $(G T)$ data take into account the fact that acoustic energy was not emitted locally, but through the speaker membrane surface, also the two-way speaker employed has a structure composed of two speakers (low tone and high tone). The crossover frequency was $3 \mathrm{kHz}$ (Genelec Studio Monitor, 2016). In practice, this means that the acoustic energy was emitted from the plane limited to the dimensions and the position of the component speakers used. In addition, Table 1 shows a boundary of acoustic potential energy emission resulting from the physical dimensions of the speakers with respect to the sound sensor. The last row of the table contains information about the relative interval of the emission signal angles $\left(\alpha_{\text {Lim }}\right)$.

Taking into consideration the presented assumptions, the sound source localization accuracy $\left(\alpha_{\text {err }}\right)$ was defined as a difference between the computed direction of arrival for the given sensor $\left(\alpha_{S}\right)$ angle and the real position of the sound source $\left(\alpha_{G T}\right)$. This parameter value is given by Eq. (10):

$$
\alpha_{\mathrm{err}}=\alpha_{\mathrm{S}}-\alpha_{\mathrm{GT}} \text {. }
$$

The examination of the localization accuracy was performed for all sensors, test signals and speakers.

During the measurements, signals of tonal and noise characteristics were used. A tonal signal was a sine waveform of variable frequency in the range of $100 \mathrm{~Hz}$ to $10.000 \mathrm{~Hz}$ with frequency changing in a logarithmic manner. The second test signal was white noise. The duration of the two signals was $10 \mathrm{~s}$. The measurement procedure consisted of three-time playback of individual test signals in a sequential manner from each speaker. During the playback of the test signals, recordings of signals emitted by the individual intensity sensors were made. As a result of these recordings, employing the tested 3D SIP and USP probes, three recordings for each speaker and the type of measurement signal were obtained.

The resulting recordings were then processed using an algorithm that allows determining DOA parameters in the frequency domain (see Subsec. 2.2). The results obtained are presented in Results Section.

\section{Results}

This section presents the results of measurements of the sound direction of arrival (DOA), obtained by measuring the intensity of sound in the frequency domain. There were two types of analysis: broadband and in the function of frequency.

\subsection{Broadband results}

Broadband analysis is based on determining the mean direction of arrival of sound regardless of the frequency. According to the methodology for determining the intensity of sound in the frequency domain, as described in Software Part Section, the analysis of all recorded signals was carried out in the offline mode. Based on the momentary data, the averaged DOA (angle of azimuth and elevation) and the standard deviation values for both signal types were determined. The obtained results are shown in Table 2. Based on the known position of the sound sources (see Table 1), the angle azimuth determination and elevation errors were calculated depending on the type of the measurement signal.

Based on the results of the broadband analysis, it should be noted that for both types of the intensity sensors, relatively accurate results of determining the

Table 2. The averaged results of the broadband analysis.

\begin{tabular}{|c|c|c|c|c|c|c|c|c|c|}
\hline \multirow{2}{*}{\multicolumn{2}{|c|}{$\frac{\text { Orientation }}{\text { Speaker number }}$}} & \multicolumn{4}{|c|}{ Azimuth [deg] } & \multicolumn{4}{|c|}{ Elevation $[\mathrm{deg}]$} \\
\hline & & 1 & 2 & 3 & 4 & 1 & 2 & 3 & 4 \\
\hline \multirow{6}{*}{ 3D SIP } & Avg. Sweep & 85.2 & 58.5 & 30.4 & 1.6 & 0.0 & -9.4 & -19.7 & -28.1 \\
\hline & Std.dev. Sweep & 9.9 & 3.8 & 4.9 & 6.0 & 6.5 & 5.0 & 5.6 & 6.2 \\
\hline & Avg. Noise & 91.0 & 60.0 & 31.2 & 2.1 & 2.0 & -8.2 & -18.3 & -26.6 \\
\hline & Std.dev. Noise & 12.2 & 12.7 & 15.3 & 21.0 & 7.4 & 7.4 & 8.2 & 9.7 \\
\hline & $\alpha_{\text {err_sweep }}$ & -4.8 & -1.5 & 0.4 & 1.6 & 0.6 & 0.3 & -1.8 & -2.2 \\
\hline & $\alpha_{\text {err_noise }}$ & 1.0 & 0.0 & 1.2 & 2.1 & 2.5 & 1.6 & -0.4 & -0.6 \\
\hline \multirow{6}{*}{ USP } & Avg. Sweep & 93.8 & 62.6 & 27.8 & -3.4 & -3.8 & -12.1 & -20.0 & -23.6 \\
\hline & Std.dev. Sweep & 3.4 & 4.8 & 4.7 & 3.9 & 2.9 & 3.0 & 3.4 & 4.4 \\
\hline & Avg. Noise & 92.1 & 64.0 & 28.3 & -5.1 & -3.4 & -11.2 & -19.4 & -23.1 \\
\hline & Std.dev. Noise & 20.9 & 19.5 & 22.9 & 26.2 & 7.0 & 7.6 & 9.5 & 10.7 \\
\hline & $\alpha_{\text {err_sweep }}$ & 3.8 & 2.6 & -2.2 & -3.4 & -2.1 & -1.2 & -1.0 & 3.2 \\
\hline & $\alpha_{\text {err_noise }}$ & 2.1 & 4.0 & -1.7 & -5.1 & -1.8 & -0.4 & -0.4 & 3.7 \\
\hline
\end{tabular}


sound direction of arrival were obtained. The location error values, calculated according to the formula (8), in the majority of cases are within the ranges of angles, where the emission of acoustic energy occurs. Error values beyond the calculated interval are shown in bold. For the 3D SIP sensor, the excess of the range of emissions occurred twice for the sweep type of the test signal (speaker 1 - azimuth and speaker 4 - elevation), and once for the measurement using white noise (Speaker 1 - elevation). For the USP sensor, the location error values in excess of the range of emissions occur more frequently: sweep signal type for speakers 1 and 4 for both the azimuth angle and elevation. For white noise, the excess was observed for the speaker 2 in the azimuth plane and the speaker 3 for azimuth and the elevation angle.

For the tonal signal, a relatively small standard deviation was obtained, while these values are lower for the USP sensor for each speaker position (except for the speaker 2 in the horizontal orientation). For the speaker 1, for the azimuth angle, the standard deviation value of the $3 \mathrm{D}$ SIP sensor is the greatest. For this speaker, the gap between the azimuth angle values ob- tained for the sweep signal type and white noise is the greatest. This fact results from the wide range of angles in a frequency range of $300-1000 \mathrm{~Hz}$ observed for the speaker. This fact has thoroughly been commented in the next Section presenting the results of the sound source localization in the function of the frequency.

On the basis of the recorded test signals, histograms of directions of sound arrival in the azimuth plane were calculated (Fig. 7 for the sweep signal and Fig. 8 for the noise boost) and elevation (Fig. 9 for the sweep signal and Fig. 10 for the noise boost). The resulting distribution is presented in the form of polar diagrams. The colors of the waveforms refer to the speakers in Figs. 5 and 6 (blue - speaker 1, green speaker 2, red - speaker 3, sea blue - speaker 4). Figure 7 shows the results in the azimuth plane. The left graph shows the results obtained for the 3D SIP probe, the right one for the USP sensor. Charts presented have not undergone any additional processing (e.g. smoothing by moving average) or e.g. equalization with Tikhonov regularization as shown in a study of GaUthier et al. (2015). It should however be noted that the use of additional processing of the final results
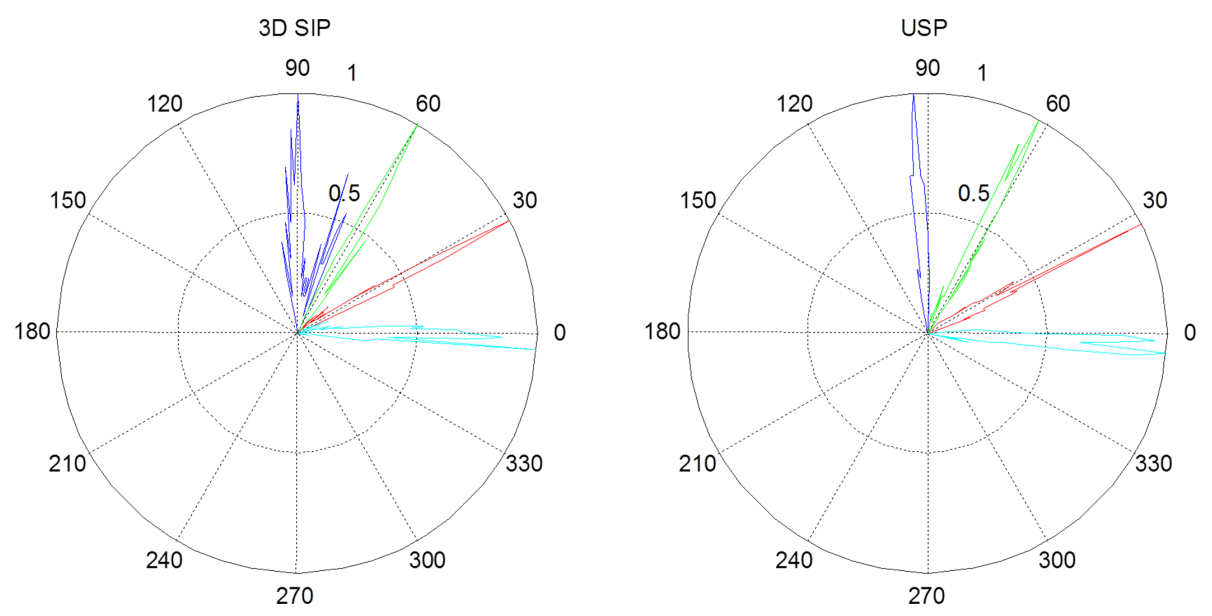

Fig. 7. Sweep signal - azimuth.
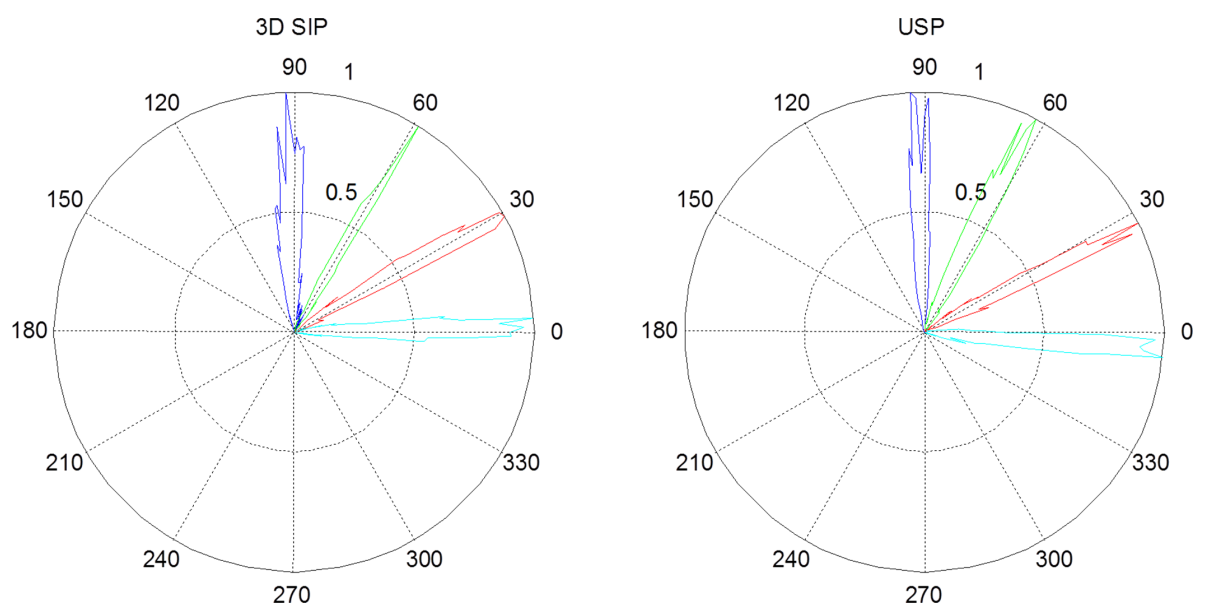

Fig. 8. White noise - azimuth. 



Fig. 9. Sweep signal - elevation.
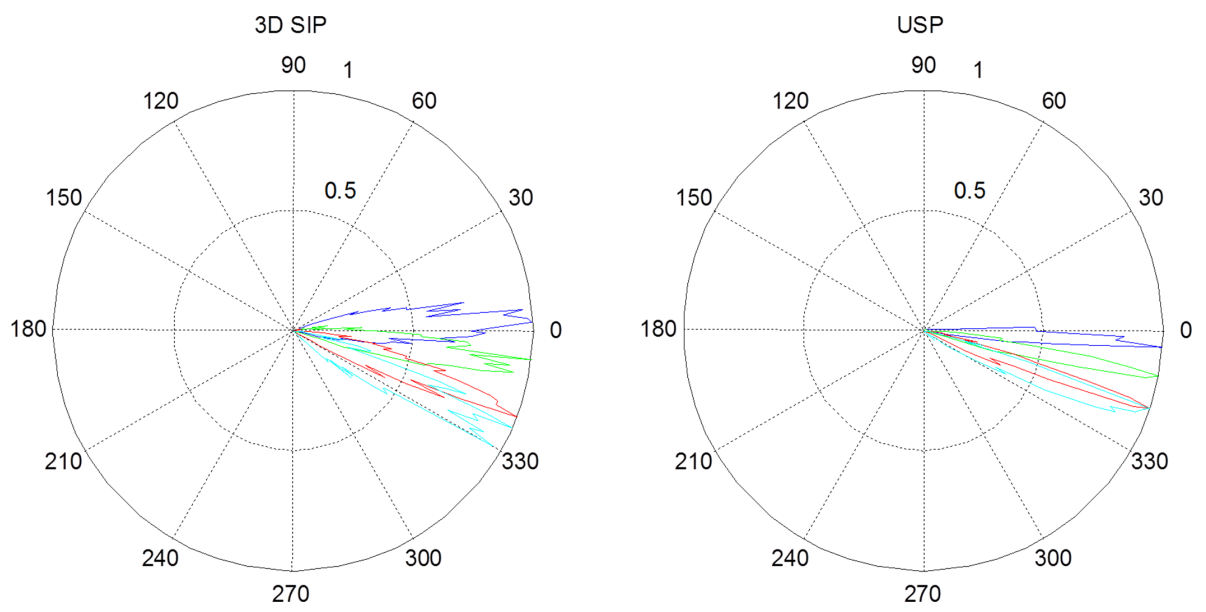

Fig. 10. White noise - elevation.

may be developed to further reduce the inaccuracy of the probe developed. The presentation of data in this form was done to show the values of the sensors themselves. In most cases, the main beams are very narrow. The exception here is the graph obtained for the 3D SIP probe sweep signal in the azimuth plane, for the speaker 1 (Fig. 7). In this case, an additional band, half the size of the main one, is clearly visible. This is due to the inaccuracy of the sensor in the low frequency range. Additional details related to this are presented in the next Section. For the noise boost, the main band is much wider compared to the distribution obtained for the sweep signal type. Waveforms for individual sensors and sound sources are very similar.

In Figs. 9 and 10, histograms of directions of arrival of sound are presented with regard to the signal used: the sweep type signal and white noise. For the USP probes, the distribution concentration in the range of 330-0 deg angles is easily to be observed (in the previous scale: $-30-0 \mathrm{deg})$. The $3 \mathrm{D}$ SIP probe waveforms are distributed more evenly, which is consistent with the actual location of speakers determined during the study. For the 3D SIP probe, the individual distributions are better separated from each other. For the
USP probes, the waveforms for speakers 3 and 4 seem to overlap. This is especially true for white noise boost (Fig. 10). This means that in the completed measuring scenario, for the elevation plane, the 3D SIP probe has better discrimination of the sound direction of arrival compared to the USP type probe.

\subsection{Frequency-dependent results}

The last type of analysis made in the course of the described research was to determine the distribution of the sound directions of arrival in the function of frequency. Designated characteristics for the sensors considered and the applied test signals are presented in Figs. 11-14. Figures 11 and 12 relate to the emission in the azimuth plane, while Figs. 13 and 14 correspond to the emission in the elevation plane, respectively for the sweep signal type and white noise. The results obtained with this type of analysis reveal the fundamental limitation of the developed $p-p$ probes, showing that there is much larger sound source location error in the low frequency compared to the USP sensor. Physical causes of these limitations are described in Sec. 1 and are presented graphically in Fig. 1. It should be 

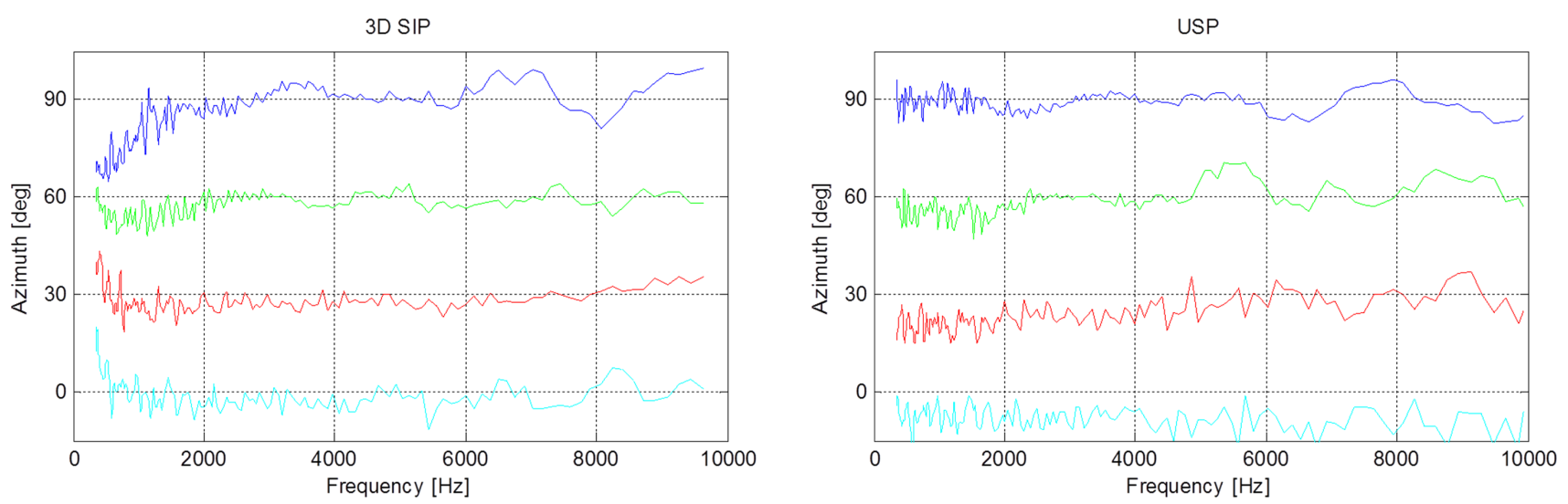

Fig. 11. Sweep signal - azimuth.

3D SIP
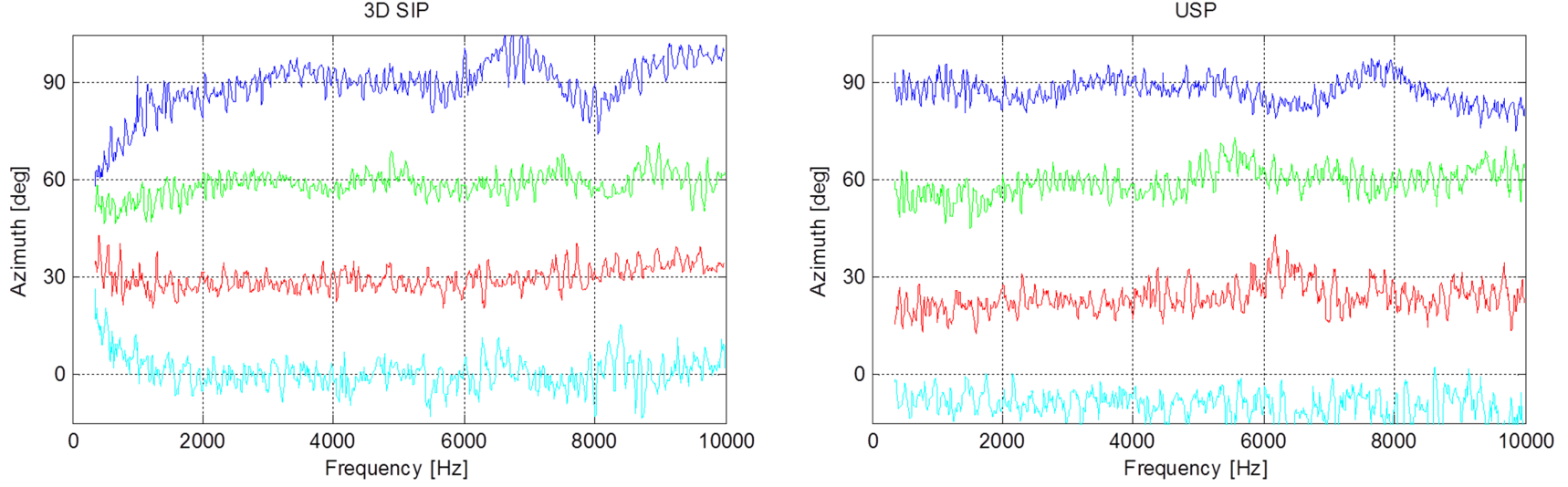

Fig. 12. White noise - azimuth.


Fig. 13. Sweep signal - elevation.
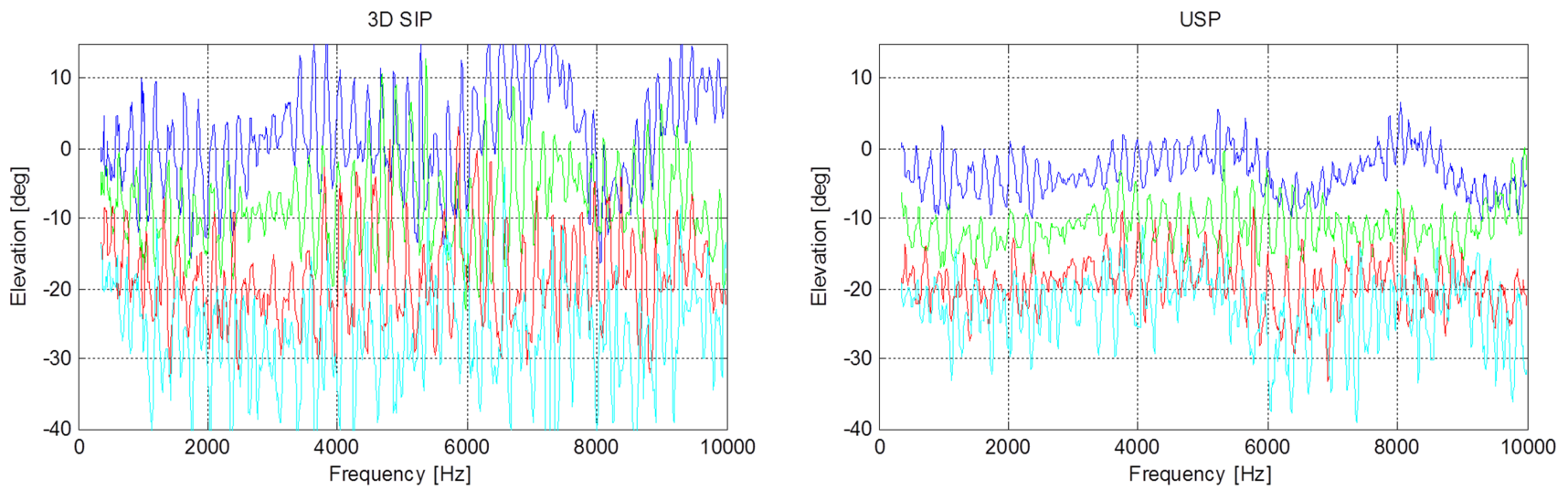

Fig. 14. White noise - elevation. 
noted that the size of the dispersion of the probe location results for 3D SIP depends on the position of the sound source. The smallest dispersion is observed for the source placed in position 2 (60 deg). There may be several causes of the observed errors in the low frequency range. The first is the variation of the parameters of the microphones constituting the individual $p-p$ pairs. During the calibration process it was disclosed that the performance of the sensitivity correction for the pair of microphones used for estimating the particle velocity in $\mathrm{OY}$ axis direction is significantly different in shape from the theoretical curve resulting from a change in wavelength (top graph in Figs. 1 and 4, green color curves), in particular in the low frequency range. The consequence of the microphones mismatch is the decrease in accuracy in the low frequency range.

The second cause of error in the low frequency range is the level of microphone own noise, which can play an important role in the situation of a small difference in signal between the microphones. In this case, the ratio of signal to noise drastically decreases, resulting in the increased dispersion of the results. For higher frequencies (greater than $1000 \mathrm{~Hz}$ ), the presented 3D SIP probe works properly.

For the USP probes, increased dispersion of results in the low frequency range was not observed. This, in turn, is the primary advantage of this type of probe. Despite its very small size, it enables an accurate measurement of the sound direction of arrival in the low frequency range.

The results obtained for the emission in the elevation plane revealed significantly better discrimination of direction of arrival of the acoustic wave for the 3D SIP probe, regardless of the frequency. This fact was also observed in the previous statistical analysis based on the DOA histogram. For the USP probe, the narrowing of resolution is clearly visible, in particular for emission of signals by speakers 3 and 4 . Characteristics obtained for the noise boost have much greater dispersion of results than is the case of tonal signals. This is confirmed by higher values of standard deviation presented in Table 2.

\section{Conclusion}

In this paper, the theoretical aspects of operation of the $p-p$ intensity probe are presented. In particular, the change in the probe sensitivity in a function of frequency is indicated, and the correction method is presented, which allows a significant improvement in the functionality of this type of a probe. Further, the design and practical implementation of a miniature $p-p$ probe operating in three directions simultaneously are presented. The method to calibrate the probe along with the algorithm for determining the direction of arrival of acoustic wave in the frequency domain were presented. The calibrated 3D SIP probe was then subjected to comparative testing. As a reference probe, commercially available USP type sensor was used. Based on numerous analyses, it was shown that the developed probe cooperatively with the digital amplitude and phase correction unit provides results comparable to the significantly more expensive solution. Moreover, in the elevation plane, much better direction discrimination was obtained than with the use of the reference sensor.

The deficiency of the presented sensor includes a tedious task of selecting the characteristics of microphones forming a $p-p$ pair. It was shown that discrepancies in microphone parameters may lead to abnormal particle velocity determination, which in turn leads to the decrease in accuracy, especially in the low frequency range. Errors of this kind only confirm the correctness of the assumptions and methodology used for calibration and amplitude-phase correction.

An important advantage of the presented solution is very low unit cost of acoustic sensors production, the functionality of which at the adopted frequency range stands up to much more expensive solutions. The possibility to produce properly functioning probes to measure the spatial distribution of the intensity of sound with the use of economical components can make the measurement technique based on the sound intensity parameter more common.

\section{Acknowledgments}

The project was funded by the National Science Centre on the basis of the decision number DEC2012/05/B/ST7/02151.

\section{References}

1. Aguilar J.R. (2015), Gunshot Detection Systems in Civilian Law Enforcement, J. Audio Eng. Soc., 63, 4, 280-291, http://dx.doi.org/10.17743/jaes.2015.0020.

2. ANSI (American National Standards Institute) S1.91996 Instruments for the Measurement of Sound Intensity (1996).

3. Cengarle G., Mateos T. (2011), Comparison of Anemometric Probe and Tetrahedral Microphones for Sound Intensity Measurements, 130th Audio Eng. Soc. Convention, May 13-16, 2011, Paper No. 8363, London, UK.

4. Cengarle G., Mateos T., Bonsi D. (2011), A Second-Order Ambisonics Device Using Velocity Transducers, J. Audio Eng. Soc., 59, 9, 656-668.

5. COAX Studio Monitor, (2016), http://apscompany.com/en/products/coax, (access 04.2016).

6. De Bree H.-E. (2003), The Microflown: an acoustic particle velocity sensor, Acoust. Aust., 31, 3, 91-94.

7. Fану F.J. (1995), Sound intensity, E \& F.N. Spon. 
8. Gauthier P.-A., Camier C., Padois T., Pasco Y., Berry A. (2015), Sound Field Repro-duction of Real Flight, Recordings in Aircraft Cabin Mock-Up, J. Audio Eng. Soc., 63, 1/2, 6-20.

9. GENELEC Studio Monitor (2016), http://www.genelec.com/support-technology/previous-models/6010astudio-monitor (access 04.2016).

10. IEC (International Electrotechnical Commission) 1043 Electroacoustics - Instruments for the Measurement of Sound Intensity - Measurements with Pairs of Pressure Sensing Microphones (1993).

11. Jacobsen F. (2008), Handbook of Signal Processing in Acoustics, pp. 1109-1127, Intensity Techniques, Springer, New York.

12. Jacobsen F. (2011), Sound Intensity and its Measurement and Applications, Acoustic Technology, Department of Electrical Engineering Technical University of Denmark.

13. Kotus J. (2015), Multiple Sound Sources Localization in Free Field Using Acoustic Vector Sensor, Multimedia Tools and Applications, 74, 12, 4235-4251, DOI: $10.1007 / \mathrm{s} 11042-013-1549-\mathrm{y}$.

14. Kotus J., Kostek B. (2015), Measurements and Visualization of Sound Intensity Around the Human Head in Free Field Using Acoustic Vector Sensor, J. Audio Eng. Soc., 63, 1/2, 99-109, DOI: 10.17743/jaes.2015.0009.

15. Kotus J., Odya P., Kostek B. (2015a), Measurements and visualization of sound field distribution around organ pipe, Proceedings of the 19th IEEE Conference SPA 2015, Signal Processing: Algorithms, Architectures, Arrangements, and Applications, pp. 145150, Poznań.
16. Kotus J., Odya P., Szczodrak M., Kostek B. (2015b), 3D Sound Intensity Measurement Around Organ Pipes Using Acoustic Vector Sensors, [in:] Progress of Acoustics, Opieliński K.J. [Ed.], pp. 105-117, Polish Acoustical Society, Wrocław Davison, Wrocław.

17. MAYA Sound Card, (2016), http://www.esiaudio.com/products/maya44usb/ (access: 04.2016).

18. Merimaa J., Lokki T., Peltonen T., KarjalaiNEN M. (2001), Measurement, Analysis, and Visualization of Directional Room Responses, 111 AES Convention, New York, USA.

19. Stigler S.M. (1974). Gergonne's 1815 paper on the design and analysis of polynomial regression experiments, Historia Mathematica, 1, 4, 431-439, DOI:10.1016/0315-0860(74)90033-0.

20. Tervo S., PÄtynen J., Kaplanis N., Lydolf M., BECH S., LOKKI T. (2015), Spatial Analysis and Synthesis of Car Audio System and Car Cabin Acoustics with a Compact Microphone Array, J. Audio Eng. Soc., 63, 11, 914-925, DOI: http://dx.doi.org/10.17743/jaes.2015.0080.

21. Weyna S. (2003), Identification of Reflection and Scattering Effects in Real Acoustic Flow Field, Archives of Acoustics, 28, 3, 191-203.

22. Weyna S. (2010), An Acoustics Intensity Based Investigation of the Energy Flow Over the Barriers, Acta Physica Polonica A., No. 1, Acoustic and Biomedical Engineering, vol. 118, pp. 172-178.

23. Woszczyk W., Iwaki M., Sugimoto T., Ono K., DE Bree H.-E. (2007), Anechoic Measurements of Particle-Velocity Probes Compared to Pressure Gradient and Pressure Microphones, 122 Audio Eng. Soc. Convention, May 2007, Paper Number: 7107. 